\title{
Remerciements aux relecteurs 2018
}

\section{Thanks to 2018 reviewers}

La revue Cahiers Agricultures remercie tous les relecteurs qui ont bien voulu l'aider à prendre une décision sur la publication des textes et ont fourni aux auteurs de précieux conseils.

Hichem Amichi

Véronique Ancey

Nadine Andrieu

Alexis J Annes

Magali Aubert

Christine Aubry

Denis Baize

Dominique Barjolle

Didier Bazile

Yves Bertheau

Thomas Bierschenk

Céline Bignebat

Genowefa Blundo Canto

Florence Brondeau

Rémi Cardinael

François Casabianca

Philippe Cattan

Leila Chakroun

Pascal Chevalier

Eduardo Chia

Nathalie Cialdella

Daniele Clavel

Anne-Laure Collard

Xavier Coquil

Nathalie Couix

Sylvie Cournut

Catherine Darrot

Hubert Ducroquet

Patrick Dugué

Céline Dutilly

Thierry Ferré

Laura Ferreira
Michel Fok

Lassina Fondio

Cédric Gaillard

Delphine Gallaud

Ariane Gaunand

Pierre Gautreau

Emmanuel Geoffriau

Allessandra Giannini

Catia Grisa

Hervé Guibert

Etienne Hainzelin

Tarik Hartani

Marie Houdart

Jean-Yves Jamin

Philippe Jeanneaux

Francis Lalöe

Dominique Louppe

Eric Malezieux

Pascal Marnotte

Enrique A Martínez

Armelle Mazé

Romain Melot

Isabelle Michel

Thierry Michels

Geneviève Michon

Marc Moraine

Thierry Morvan

Claire Mouquet-Rivier

Mireille Navarrete

Guillermo Neiman

Ruth Nettle

Aboubakar Njoya
Brigitte Nougarèdes

Tom Osborn

Marc Oswald

Harry Ozier-Lafontaine

Elise Pelzer

Coline Perrin

Jean-Pascal Pichot

François Piraux

Etienne Polge

Ismael Ràfols

Jean Ch. Randriamboavonjy

Valery Rasplus

Christine Rawski

Alexis Roy

Eric Sabourin

Nicolas Salliou

Hilmy Sally

Elsa Sanial

Babacar Sène

Nacro Souleymane

Mohamed Taher Sraïri

Thibault Sterckeman

Gabin Tarrouth

Ludovic Temple

Pierre Triboulet

Gilles Trouche

Elodie Valette

Eric Vall

Philippe Vernier

Betty Wampfler

Nadine Zakhia-Rozis

Sibiri Jean Zoundi 